\title{
Numerical analysis of PCM melting filling a rectangular cavity with horizontal partial fins
}

\author{
Tarik Bouhal ${ }^{1,2^{*}}$, Saïf ed-Dîn Fertahi ${ }^{2}$, Oussama Limouri ${ }^{l}$, Younes Agrouaz ${ }^{1,2}$, Tarik Kousksou ${ }^{l}$, Youssef Zeraouli ${ }^{1}$, \\ Abdelmajid. Jamil ${ }^{2}$
}

${ }^{1}$ Université de Pau et des Pays de l'Adour (UPPA/E2S), Laboratoire des Sciences pour l'Ingénieur Appliquées à la Mécanique et au Génie Electrique (SIAME) - Fédération IPRA, EA4581, 64000, Pau, France

${ }^{2}$ Université Sidi Mohammed Ben Abdellah (USMBA), École Supérieure de Technologie de Fès, Route d'Imouzzer, BP 2427, Fez, Morocco

\begin{abstract}
The present numerical study is conducted to analyze melting process within a rectangular enclosure filled by phase change material (PCM) vertically heated from one side. The right hot wall and the left cold wall are maintained at temperatures, $\mathrm{T}_{\mathrm{h}}=38.3^{\circ} \mathrm{C}$ and $\mathrm{T}_{\mathrm{c}}=28.3{ }^{\circ} \mathrm{C}$, respectively, and it was filled by solid PCM Gallium initially at temperature $\mathrm{T}_{\mathrm{c}}$. The horizontal walls are insulated. A transient numerical model is developed to study the heat transfer and melting behaviours, and the natural convection is accounted. To enhance the heat transfer and the melting process of the PCM, fins with a rectangular and triangular shape are proposed. Moreover, the effects of both thermophysical properties and fins integration on the flow structure and heat transfer characteristics are investigated in detail. The melt fraction contours with the natural convection driven flow are performed and compared, as well as the temperature distributions for a Rayleigh number of around $\mathrm{Ra}=10^{6}$. It is found that the rate of the melting increases with the elevation in the values of specific heat capacity $\mathbf{C}_{\mathbf{p}}$ as well as the thermal conductivity $\boldsymbol{\lambda}$ of the PCM Gallium. The results show also that the rectangular fin accelerates the PCM melting faster than the triangular fin's shape thanks to the increased exchange area.
\end{abstract}

\section{Introduction}

Nowadays, thermal energy storage improvement using phase change materials (PCMs) has become a highly central issue of major importance in the scientific research community [1]. Particularly, melting coupled with natural convection in enclosures containing a PCM has been the subject of many experimental and numerical studies due its wide area of interest in several fields such as passive cooling of electrical or electronic components, thermal insulation and latent energy storage $[2,3]$. The effectiveness of cooling devices using PCM integrated in electrical components depends on the melting speed of the PCM so that the latent heat plays its role rapidly but also over time [4]. However, there are few studies in the literature reports including fins integration of PCMs, which have significant potential to enhance the thermal performance of PCMs. In fact, the main disadvantage of PCMs is their low thermal conductivities. Researchers considered different ideas to improve heat transfer between the PCMs and a particular boundary or fluid such as extending surfaces using fins [5]. In this paper, two-dimensional CFD simulations were performed to simulate the melting process of a phase change material (PCM) filling a rectangular cavity which includes fins. A CFD model based on the physical enthalpy-porosity formulation was used to simulate the phase change of the solid Gallium and to optimize the melting time of the used PCM. The geometric effect of the fins, as well as the thermophysical properties on the heat transfer characteristics are investigated in detail. The results are presented in terms of isotherms, liquid fraction and local temperatures evolution. The CFD software OpenFoam was used in order to carry out the numerical simulations and also to explain the numerical observations and results.

\section{Analysis and modelling}

In the present paper, we have conducted a numerical investigation on a rectangular enclosure filled by a solid PCM. The dimension of the cavity used in this work are $\mathrm{L}=8.89 \mathrm{~cm}$ and $\mathrm{W}=6.35 \mathrm{~cm}$ as schematically presented in Figs. 1. A single rectangular and triangular fin of $t=4$ $\mathrm{mm}$ thickness is integrated in the right wall where a hot temperature condition $\mathrm{T}_{\mathrm{h}}=38.3{ }^{\circ} \mathrm{C}$ is applied (the right and fin's walls are together isotherms) while the left wall is subjected to a cold temperature $\mathrm{T}_{\mathrm{c}}=28.3{ }^{\circ} \mathrm{C}$. The horizontal walls are maintained constantly adiabatic and the initial temperature of the $\mathrm{PCM}$ is $\mathrm{T}_{\mathrm{c}}$. A no-slip condition is imposed at the solid surface wall, i.e., the velocity of the PCM at the wall is zero.

\footnotetext{
* Corresponding author: bouhal.tarik12@gmail.com
} 

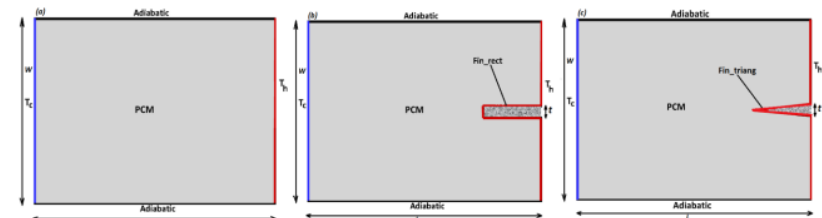

Fig. 1. Layout of the rectangular enclosure filled by PCM without fin (a), with rectangular (b) and triangular (c) fins

Three different configurations were investigated: Enclosure without fin and with rectangular or triangular fins. The boundary condition setup are presented in Figs. 1. The Figs. 2 show a structured grids depending on which rectangular cavity configuration is considered. The final mesh was composed of about 9375-12000 elements and a time step of $10^{-3} \mathrm{~s}$ and $10^{-4} \mathrm{~s}$ was found for. The parameters Lv (Vertical line), Lh (Horizontal line) and Pc (Center point) are the monitors used to describe the evolution of temperature inside the cavity (see Figs. 2).
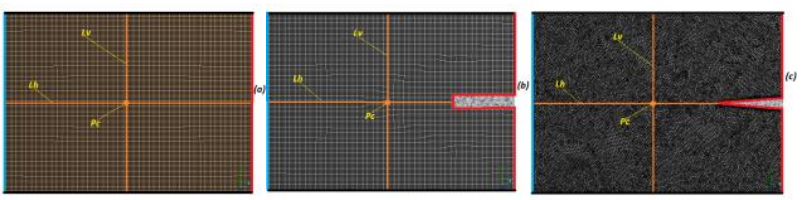

Fig. 2. Mesh grid of the rectangular cavity without fin (a), with rectangular (b) and triangular (c) fins

For the enclosure's reference case i.e. the configuration without fin, we are interested in studying the effect of the PCM's thermophysical properties on the melting process and flow structure especially the impact of thermal conductivity $\lambda$ as well as the specific heat capacity $\mathbf{C}_{\mathbf{p}}$. The values of thermophysical properties chosen for this parametric study are shown in Table 1.

Table 1. PCM's thermophysical properties chosen for the parametric study.

\begin{tabular}{|c|c|c|}
\hline Parameter & $\mathbf{C}_{\mathbf{p}}(\mathrm{J} / \mathrm{kgK})$ & $\lambda(\mathrm{W} / \mathrm{mK})$ \\
\hline Cavity without fin & $\begin{array}{c}381.5 \\
\left(\mathrm{C}_{\mathrm{pref}}\right)\end{array}$ & $32\left(\boldsymbol{\lambda}_{\text {ref }}\right)$ \\
\hline- & 300 & 28 \\
\hline- & 450 & 24 \\
\hline
\end{tabular}

Indeed, when changing the PCM thermophysical properties, the Rayleigh number has a range values in this intervals $1 \times 10^{6}<\mathrm{Ra}<3 \times 10^{6}$. The working PCM used within the rectangular cavity is the gallium. The thermophysical properties for pure gallium used in this investigation are given in Table 2 .

Table 2. Physical properties of PCM pure Gallium.

\begin{tabular}{|l|l|}
\hline Parameters & Values \\
\hline
\end{tabular}

\begin{tabular}{|c|c|}
\hline Reference density & $6095 \mathrm{~kg} / \mathrm{m}^{3}$ \\
\hline Thermal expansion coefficient & $1.210^{-4} 1 / \mathrm{K}$ \\
\hline Melting temperature & $29.78^{\circ} \mathrm{C}$ \\
\hline Reference temperature & $29.78^{\circ} \mathrm{C}$ \\
\hline Latent heat of fusion & $80160 \mathrm{~J} / \mathrm{kg}$ \\
\hline Specific heat capacity & $381.5 \mathrm{~J} / \mathrm{kgK}$ \\
\hline Dynamic viscosity & $\begin{array}{c}1.8110^{-3} \\
\mathrm{~kg} / \mathrm{m} . \mathrm{s}\end{array}$ \\
\hline Prandtl number & $2.1610^{-2}$ \\
\hline
\end{tabular}

The main objective of the present study is to investigate numerically the heat transfer during the melting process of PCM within a rectangular enclosure cavity with and without fins using the CFD platform OpenFoam (see Figs. 1). This study is motivated by the need to gain improved understanding of heat transfer during the charging phase of thermal energy storage system which takes advantage of latent heat-of-fusion of PCM. A relevant consideration in such systems is the effective utilization of the rectangular cavity containing PCM by an optimum fins topology and also highlighting the impact of the PCM's thermophysical properties on the physical phenomena such as melting process and heat transfer structure for the configuration without fin (reference). Then, a comparison study is performed between the reference configuration and the other configurations with fins.

\section{Mathematical formulation}

Unsteady two-dimensional flow models of heat transfer during the melting process of PCM inside a rectangular enclosure were governed by the general following assumptions:

-The thermophysical properties of the PCM are constant except for the density which obeys to Boussinesq approximation;

-The fluid is Newtonian and incompressible;

-The viscous dissipation is negligible;

-The fluid motion in the melt is two-dimensional and laminar characterized by a smooth streamlines and an ordered motion because $\mathrm{Ra}$ has a value around $\mathrm{Ra}=10^{6}$.

The energy and Navier-Stokes equations were used to solve the transient hydrodynamic and thermal fields. Therefore, the resulting governing equations with consideration of gravity effect can be written as follows taking into account the foregoing assumptions: 
- The energy equation in the phase change

$$
\text { zone }
$$

$$
\frac{\partial\left(\rho_{p} h\right)}{\partial t}+\nabla \cdot\left(\rho_{p} \vec{v} h\right)=\nabla \cdot\left(\lambda_{p} \nabla T\right)+S
$$

- In the energy equation :

$$
\begin{gathered}
h=h_{p}+\Delta h \quad h_{p}=h_{r e f}+\int_{T_{r e f}}^{T} C_{p} d T \quad \Delta h=\beta r_{p} \\
S=\frac{(1-\beta)^{2}}{\left(\beta^{2}+\Delta\right)} A_{m u s h}\left(v-\vec{v}_{p}\right)
\end{gathered}
$$

- The liquid fraction is defined by :

$$
\beta=\left\{\begin{aligned}
0 & \text { si } T<T_{s} \\
1 & \text { si } T>T_{l} \\
\frac{T-T_{s}}{T_{l}-T_{s}} & \text { si } T_{s}<T<T_{l}
\end{aligned}\right.
$$

- Continuity equation:

$$
\frac{\partial \rho}{\partial t}+\nabla \cdot(\rho \vec{v})=0
$$

- The equation of motion taking into account the Boussinesq approximation

$$
\frac{\partial \rho \vec{v}}{\partial t}+\nabla \cdot(\rho \vec{v} \cdot \vec{v})=-\nabla P+\nabla \cdot \bar{\tau}-\rho_{0} g \beta\left(T-T_{f}\right)
$$

\section{Numerical procedure}

As previously mentioned, the numerical modelling was conducted using the software OpenFoam to simulate the melting of PCM. The geometric model of Figs. 2 was created and meshed to solve the equations involving the melting process, namely the energy, continuity and momentum equations. Instead of following the formation of the liquid-solid front explicitly, an enthalpy-porosity formulation was used, after obtaining the enthalpy field and temperature, to characterize the phase change interface. The solution for temperature is essentially an iteration between the energy equation and the liquid fraction equation which is directly used to update the liquid fraction usually results in poor convergence of the energy equation.

\section{Results and discussion}

The numerical study investigates the melting of PCM Gallium in a rectangular enclosure of size $88.9 \mathrm{~mm} \times 63.5 \mathrm{~mm}$ with constant hot temperature from the right side and cold temperature from the left wall of the domain and other horizontal sides are perfectly insulated. The results of the simulation were recorded at regular intervals of 5 minutes for a complete melting cycle of 35 minutes. Those results are represented as temperature distribution and contours of liquid fraction in addition to Nusselt number during time.

\subsection{The characteristics of the melting process for the reference case without fin}

Fig. 3 shows the contours of temperature and the variation in temperature after regular interval of 5 minutes each. It can be observed from the Fig. 3 that the maximum temperature achieved is $302 \mathrm{~K}$ during the initial stage and $311 \mathrm{~K}$ at the later stages of the melting process. It can also be observed from Fig. 3 and 4 that, when the temperature at any location in the PCM domain reaches $303 \mathrm{~K}$ the PCM starts melting. The blue color shows the solid region. The shape of this zone resembles to that of PCM liquid fraction as shown in the Fig4. There is a red zone developing in the PCM domain nearer to the right wall after $5 \mathrm{~min}$, and it gradually increases in size. This is because of the natural convection effects present in the liquid PCM. At the end of the melting process approximately all the PCM is in liquid phase and the properties of the PCM becomes constant which can be clearly seen in Fig. 4 for $t=30$ $\min$.

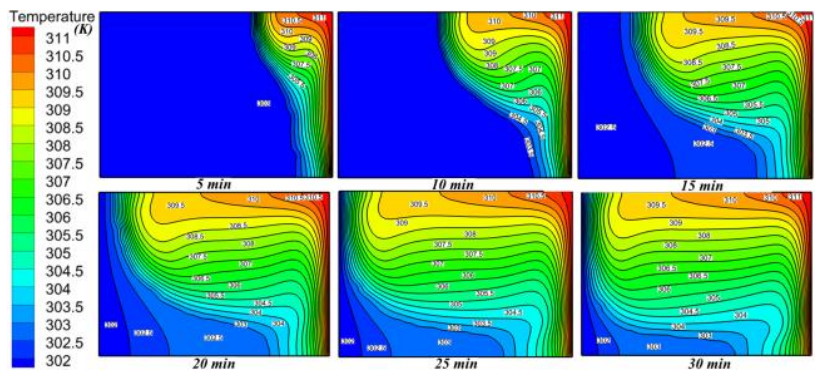

Fig. 3. Temperatures distribution of the PCM (Configuration without fin)

Fig. 4 shows the variation of liquid fraction with time. We can easily examine the shape and motion of melting interface as the time passes. The blue and red colors represent the condition when material is completely liquid $(\boldsymbol{\beta}=1)$ and solid $(\boldsymbol{\beta}=0)$, respectively. The mushy zone represents the melting front; it separates the liquid and solid region. We can observe that at the initial stages $\mathrm{t}=0-5 \mathrm{~min}$ of melting process the melting interface is almost parallel to the left wall, which indicates that in early stages the heat transfer is mainly by conduction. As the time elapsed to 10 minutes the liquid PCM having lower density and higher temperature rises up and then moves downward and this continues for a large number of cycles. It can also be seen that it takes around 25 minutes to melt the top layer of the PCM cavity. It takes around 15 minutes to melt half of the PCM in the rectangular cavity. 

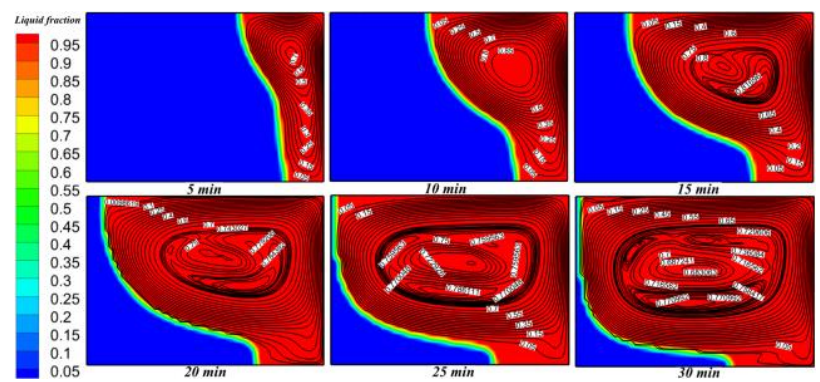

Fig. 4. Liquid fraction evolution of the PCM (Configuration without fin)

\subsection{Liquid fraction evolution and temperature distribution for the configuration without fin}

Fig. 5-(a) represents the variation of the melting fraction i.e. the percentage of the PCM which has been melted, as function of time. We can see that at $\mathrm{t}=0 \mathrm{~min}$ the melting fraction is zero because all the PCM in the domain is in solid state. As the time passes due to heating the PCM gets melted and the value of melting fraction increase with time. The value of melting fraction is $25 \%, 35 \%$, $50 \%, 70 \%$ and $90 \%$ for the melting time of $5,9,15,20$, and 30 minutes. At the end of the melting cycle i.e. after 35 minutes all the PCM in the PCM domain has been melted; giving a melting fraction of $100 \backslash \%$. It is noticed that the rate of melting is almost same at the starting of the melting process and it increases in the middle and at the end of the melting process. As presented in Fig. 5(a), the configuration with fins melted the PCM in 13.35 min, while the configuration without fins have melted it in $18.35 \mathrm{~min}$. In this case, the melting time gained due to the integration of thin fins on the circumference of the heating source is $5 \mathrm{~min}$. For Fig. 5-(b), the melting time lasts 5 min with regard to the configuration with fins. For the cylindrical configuration the melting time is extended to $10 \mathrm{~min}$. As a result, the melting time is estimated to be $5 \mathrm{~min}$. The temperature evolution of Gallium was monitored locally inside the rectangular cavity as presented in Fig. 5-(b), (c) and (d). Three monitors have been defined to measure the progression of the liquid fraction and the temperature evolution within the enclosure (see Fig. 5) during the three phases that the Gallium passes through: solid phase, mushy phase and liquid phase. In Fig. 5, the local center temperature evolution and two lines, namely Lv (Vertical line) and Lh (Horizontal line) have been presented, as they highlight the effect of the boundary conditions to the improvement of the melting time. The applied temperature in this case was $\mathrm{T}_{\mathrm{h}}=38.3^{\circ} \mathrm{C}$. It has been found that PCM takes much melting time along the horizontal line compared to vertical one.
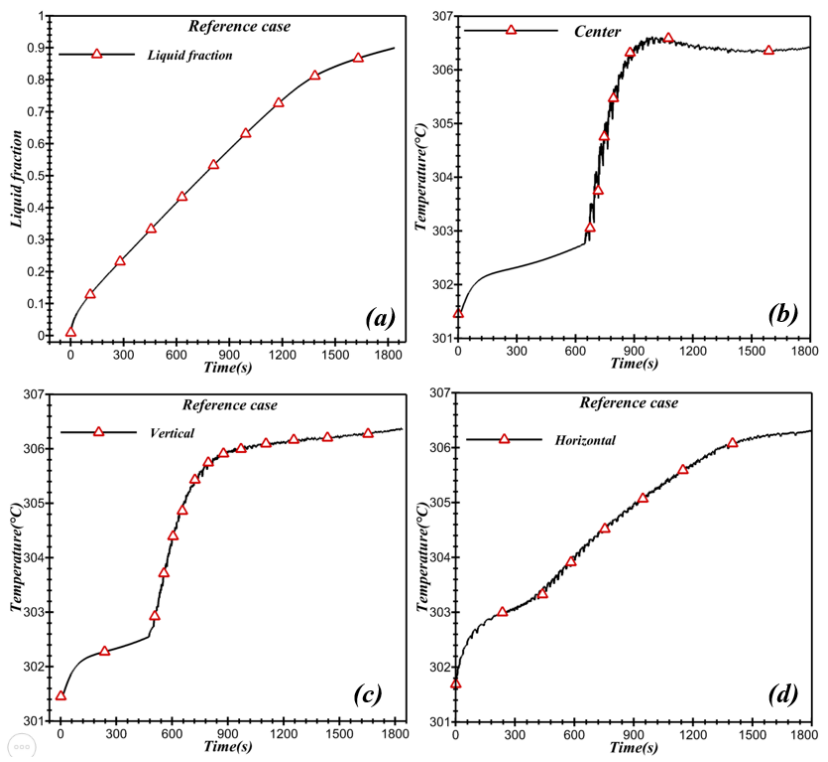

Fig. 5. Liquid fraction evolution and temperatures distribution of the PCM (Configuration without fin)

\subsection{Effect of PCM thermophysical properties on melting process}

\subsubsection{Temperature and liquid fraction contours for various $C_{p}$}

Figs. 6 show the temperature contours as well as the liquid fraction after a time of $5 \mathrm{~min}$ for different values of specific heat capacity $\mathbf{C}_{\mathbf{p}}$. The conductivity $\boldsymbol{\lambda}$ is maintained constant when the $\mathbf{C}_{\mathbf{p}}$ of the PCM varies. The thermal properties depend on the type of PCM. We note that the heat capacity clearly influences the kinetics of melting. In fact, the melting velocity increases proportionally with the increase in the heat capacity value.

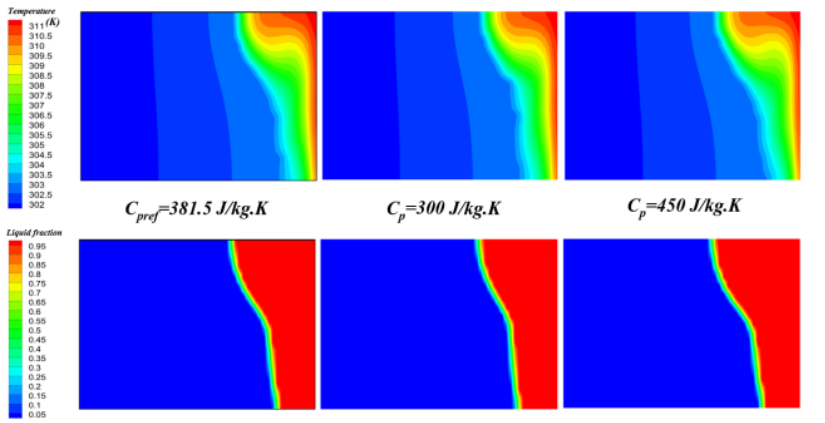

Fig. 6. Effect of $\mathbf{C}_{\mathbf{p}}$ on PCM melting process (Configuration without fin, $\mathrm{t}=5 \mathrm{~min}$ )

\subsubsection{Temperature and liquid fraction contours for various $\lambda$}

Figs. 7 represent the temperature contours as well as the liquid fraction after a time of $5 \mathrm{~min}$ for different values of thermal conductivity $\boldsymbol{\lambda}$. The $\mathbf{C}_{\mathbf{p}}$ is maintained constant when the $\lambda$ of the PCM varies. It can be noticed that the 
thermal conductivity has a slight influence on the kinetics of melting. Indeed, the melting process is affected slowly by changing the conductivity of the PCM.
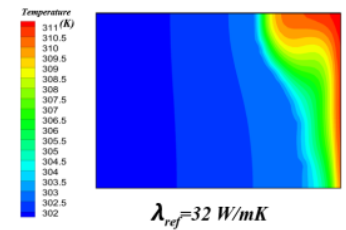

$\lambda_{r e f}=32 \mathrm{~W} / \mathrm{mK}$
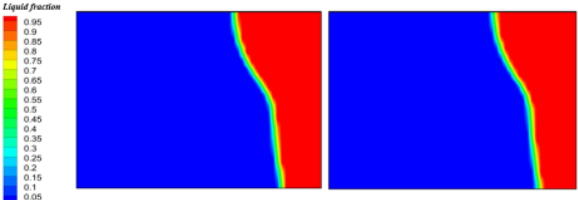

Fig. 7. Effect of $\boldsymbol{\lambda}$ on PCM melting process (Configuration without fin, $\mathrm{t}=5 \mathrm{~min}$ )

\subsection{Effect of fins integration in the enclosure}

In this part, we investigated the effect of the fin's integration in the cavity to show the kinetics of the melting front. It's interesting to note that the fins are isotherms and not just heat conducting. Therefore, the contours of the temperature and liquid fraction for the studied configurations without fin (reference case) and with rectangular and triangular fins are presented in Fig. 8. As we can notice, the fins improved the melting time. In fact, during $5 \mathrm{~min}$, an important part of the PCM has been melted for the configurations with fins compared to reference case (without fin).
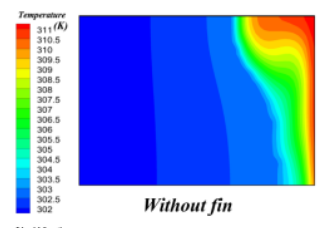

Without fin

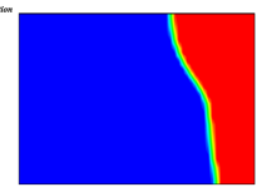

Fig. 8 Effect of fins shape integration on melting process $(\mathrm{t}=5 \mathrm{~min})$

To emphasize these observations, the evolution of the liquid fraction as a function of time for the studied configurations are presented in Fig. 9.

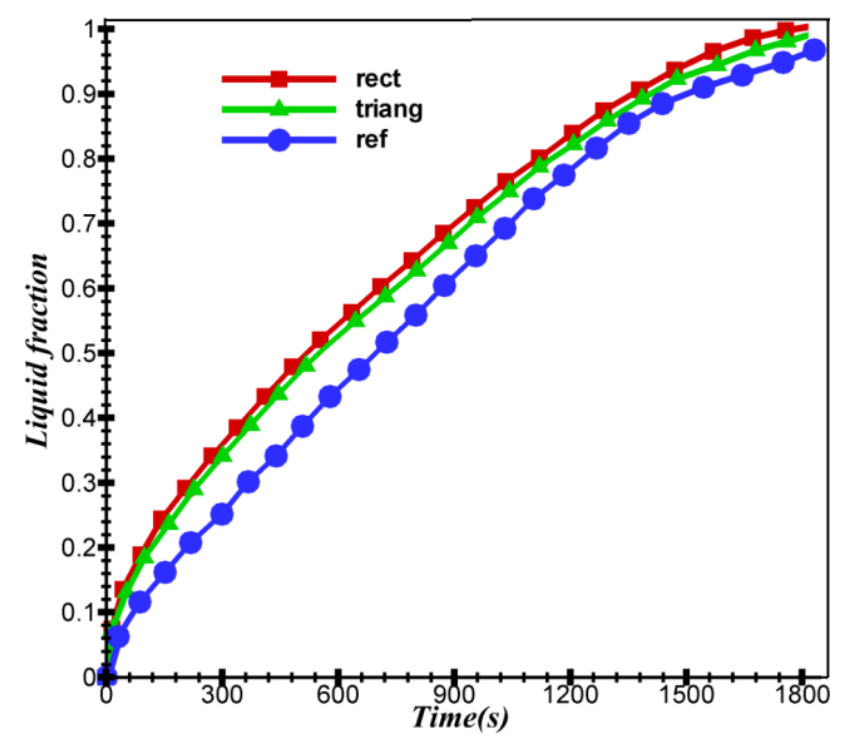

Fig. 9 Effect of fins geometries integration on melting process

Obviously, we notice that the integration of fins and their shapes have a strong influence on the kinetics of melting. Indeed, the rectangular shape accelerates fastly the melting time more than the triangular one. This is due to the exchange surface which promotes convection thanks to the large surface for the case of the rectangular fin. Since the PCM has a low conductivity, it seems that the fin integration has a primordial role in promoting the heat transfer phenomena and accelerating the PCM melting time.

\section{Conclusion}

The melting process along a rectangular enclosure filled by a PCM (Gallium), where two shape's fins were included, has been performed through this paper using two-dimensional CFD simulations. In fact, three configurations were examined, the first configuration without fin, and both configurations with a rectangular and triangular thin fins integrated in the middle of the right hot enclosure's wall. The parametric studies include also the effect of thermophysical properties essentially $\mathbf{C}_{\mathbf{p}}$ and $\lambda$ on the melting process and on the physical phenomena. The results show that integrating fins in the enclosure wall have enhanced the heat transfer in the PCM located inside the rectangular cavity and improved its melting time while the applied a hot temperature $\left(\mathrm{T}_{\mathrm{h}}=38.3^{\circ} \mathrm{C}\right)$. A continuation of this work will concern the effect of other PCM properties and the study of other fin's shape, locations and orientations.

The authors are thankful to the "Institut de Recherche en Energie Solaire et Energie Nouvelles" (IRESEN-Morocco) for the financing support under the project of Solar Cooling Process in Morocco (SCPM).

\section{References}

1. Mat, S., Al-Abidi, A.A., Sopian, K., Sulaiman, M.Y., Mohammad, A.T., 2013. Enhance heat transfer for PCM melting in triplex tube with internal-external fins. Energy conversion and management 74, 223-236. 
2. Kousksou, T., Bruel, P., Cherreau, G., Leousso, V., El Rhafiki, T., 2011. PCM storage for solar DHW: From an unfulfilled promise to a real benefit. Solar Energy 85, 2033-2040.

3. Kabeel, A., Teamah, M.A., Abdelgaied, M., Aziz, G.B.A., 2017. Modified pyramid solar still with vcorrugated absorber plate and PCM as a thermal storage medium. Journal of Cleaner Production.

4. Kousksou, T., Mahdaoui, M., Hlimi, M., El Alaiji, R., El Rhafiki, T., 2016. Latent energy storage: Melting process around heating cylinders. Case Studies in Thermal Engineering 8, 128-140.

5. Ji, C., Qin, Z., Low, Z., Dubey, S., Choo, F.H., Duan, F., 2018. Non-uniform heat transfer suppression to enhance PCM melting by angled fins. Applied Thermal Engineering 129, 269-279. 